\title{
Deployment of the health education program in a healthy city
}

\author{
Miyoko Hamano ${ }^{1}$ and Junko Watanabe ${ }^{2}$ \\ ${ }^{1}$ Tokyo Kasei-Gakuin University and ${ }^{2}$ Tokyo Medical College
}

\begin{abstract}
It is important to go ahead with the plan which combines local communities with concerned individuals and to work together in an effort to accomplish community-based positive health. In this article, the health education program maintains an objective of positive health of women of middle or advanced-age. Planning, operation and evaluation were used to develop an empowerment technique. Women of positive health class were admitted based on body composition, energy and blood lipid item. They appeared to be most interested in the motivation of thinking and continuing the execution of improvement of their lifestyles. It has been determined that the academic board which employed epidemiologic method bringing long term continuing positive effect and QOL into view is important, in order to carry on better estimate and remediation of education.
\end{abstract}

Key words: Health education program, empowerment, middle-aged woman, positive health, Health behavior

\section{Purpose}

The most important health issue of long-lived society is to extend healthy life expectancy. Japan's average life and healthy life expectancy beat the rest of the world according to WHO (2005) ${ }^{1)}$ and responsiveness ${ }^{2}$ ) which contends that quality of medical treatment takes seventh place. Comprehensive health achievement including these items is estimated to be the best in the world. On the other hand, fair social-security burdens such as medical bills are expected to make aged society healthy and lively. Furthermore, decrease of exercise, unbalanced diet and decline of physical strength are problems in daily life. The purpose of positive health changed to "exalting QOL and living longer" form "living a long life" and it aims to build a society in which each citizen can live bravely and actively through one's lifetime. It is difficult to accomplish and maintain on one's own, so we need to focus on finding a plan which combines local communities with each concerned individual in order to reach this goal. In order to make health education more effective in our advanced research $^{3)}$ since 1989, authors introduced an empowerment technique ${ }^{4)}$ 5) 6) to develop their plan instead of to teach them as education by the local-community. To analyze participants' health activity objectively, awareness of independent and specific health behavior such as "protect the health of one's own" "7) was enhanced. In this report, learn-by-doing teaching whose purpose is to clarify the effects of user of the community health center continuing "positive health class" on transformation of their lives.

\section{Method}

(1) The empowerment technique; by approaching the education program through a process of commitment, dialogue, building up issue and sense of fellowship and action, the pillar of the strategy important for training of talented people or the production of an organization was able to be built. The key point of the plan is to promote development of human resource and organization. Also, the health educations which promote empowerment attempt the continual assistance by interlocking each level such as individual, organization and community.

(2) Subjects: 28 women (average age 37 to 61 ) who went thorough the national based health check-up at Shiroyama-Cyo, Tukui-gun, and Kanagawa. Subjects learned at "positive health class" with a focus on assistance of expert staff for six months, and after a year, they attend learning in practice for education program three times in a row.

(3) The protocol of learning: At Positive health class, exercise training, nutrition training and lifestyle guidance are carried out after a medical check on the course at once per week for 3 months 
for a total of 12 times. According to subjects; physical characteristic, BP and blood lipid item were recorded before they attended the class, 12 of them were hyperlipemia or obesity restricted respectively. This learning program's consideration is the factor which relating lifestyle habits such as smoking, drinking, exercise habit, nutrition condition, physical strength and blood lipid item.

\section{A result and consideration}

\section{3-1. The issue and approach aim for "The health city"}

In Shiroyama-Cho, the health plan that was developed in 1989 aimed for the establishment of a system of total health based on local-community by cooperating with health, medical and welfare systems. In that case, the university participated in this health plan as part of local contribution, and has continued till today. By carrying out the health plan, the problems such as (i) shortness of healthy life expectancy, (ii) higher medical bill than other communities, and (iii) citizens' dissatisfaction with services became clear.

According to the result, in order to "protect the health of one's own", "decrease of lifestyle related diseases death rate" must be accomplished. Therefore to place importance on the education after a medical checkup for preemptive care, we focused on the strengthening of present business. It became as improvement goal. To accomplish the goal, not only an expert staff but also local resource network's efficiency was improved and cooperating system were developed. ${ }^{8),} 9$ ), 10)

Table 1 Participant's healthy learning program

\begin{tabular}{|c|c|c|c|}
\hline & A participant's study activities & $\begin{array}{l}\text { The contents of a } \\
\text { classroom }\end{array}$ & Study support from the staff \\
\hline $\begin{array}{l}\overrightarrow{+} \\
\stackrel{\grave{\Xi}}{\bar{D}}\end{array}$ & $\begin{array}{l}\text { - The talks which notice the problem } \\
\text { of their area and look for corrective } \\
\text { strategy } \\
\text { - Its life action improvement target for } \\
\text { one month is set up, and the degree } \\
\text { of achievement is predicted. }\end{array}$ & $\begin{array}{l}\text { He notices his } \\
\text { problem and a } \\
\text { target is decided } \\
\text { for an } \\
\text { improvement. }\end{array}$ & $\begin{array}{l}\text { - It explains as a result of a medical } \\
\text { checkup and question paper } \\
\text { investigation. } \\
\text { - The role of a moderator of talks }\end{array}$ \\
\hline 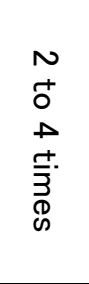 & $\begin{array}{l}\text {-Self-inspection of meal record } \\
\text { - The design for the meal suitable } \\
\text { for a life style } \\
\text { - Self-valuation of a menu }\end{array}$ & $\begin{array}{l}\text { - It learns about the } \\
\text { balance of the meal } \\
\text { suitable for its } \\
\text { health and life } \\
\text { style. }\end{array}$ & $\begin{array}{l}\text { - A program is corrected so that a } \\
\text { question of a participant may be } \\
\text { incorporated in the next } \\
\text { contents. } \\
\text { The role assignment to a } \\
\text { participant }\end{array}$ \\
\hline $\begin{array}{c}5 \\
\text { Time }\end{array}$ & - Practical skill & $\begin{array}{l}\text { - The knowledge about } \\
\text { movement and } \\
\text { practical skill suitable } \\
\text { for their own body } \\
\text { are mastered. }\end{array}$ & $\begin{array}{l}\text { - A lecture and practical skill } \\
\text { instruction } \\
\text { - It corresponds to the question } \\
\text { from a participant with practical } \\
\text { skill instruction. }\end{array}$ \\
\hline $\begin{array}{c}6 \\
\text { Time }\end{array}$ & $\begin{array}{l}\text {-Self-valuation of achievement of } \\
\text { the } 1 \text { st target }\end{array}$ & & \\
\hline
\end{tabular}

\section{3-2. Deployment and the learning program of health education}

Future-oriented health welfare rehabilitation and bonding health service's workforce was established in 1990. Education deployment system which is always available and proactive for local residents was provided and the program became available to subjects. Subjects certificated "OB" becoming a staff made coming onsite more possible. OB was mainly relegated group work and expert staffs carrying out analysis of daily life issues. Subjects can share and rate visible changes in numeric value and carry out accuracy control; therefore efficient learning can be promoted without lowering class function ${ }^{11), 12), 13), 14)}$. Now, because of the introduction of IT, national registered dietitians have increased the impact of educational guidance because they can respond to the result of dietary examinations. 


\section{3-3. Examination of the validity in a health education "positive health class" continuation participant}

(1) Subjects

Subjects' 28 persons who went thorough the health check-up and were picked out by BMI and OB staffs, participated the health learning program as shown in table 1 for six months. They also attended the course 12 times as described above, three times in a row. In table 2, Subjects' bodily features on the first health check-up are shown. The weight shown in Fig 1 decreased each time they attended the class and they lost 1 to 7 kilograms by the last class. The average percent of body fat was determined as "slight obesity" but decreased significantly by the second class. The percent of body fat's attrition rate was great in each class. The same results were accepted in obesity patients. This time, all of subjects are housewife and about $25 \%$ of subjects were under stress. Causes of their stress are "health issue of their children", "husband's work" and "trouble between one's wife and one's mother."

Table 2 Continuation participant of production classroom of health

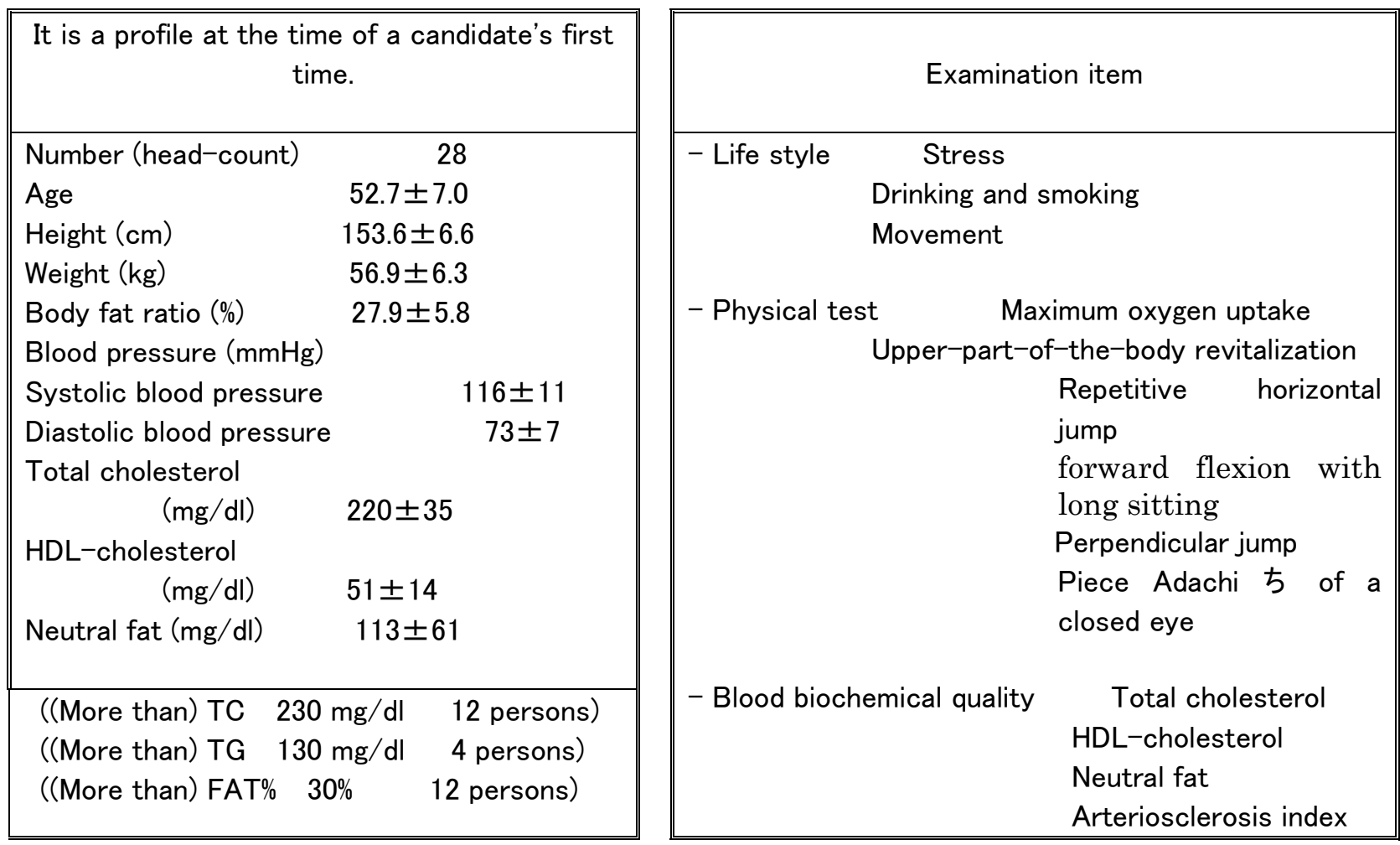

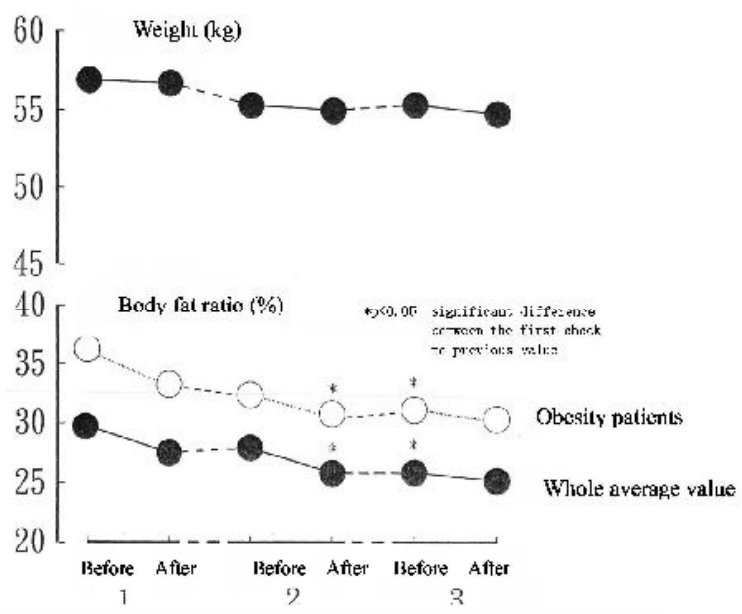

Fig. 1 Change of weight and body fat ratio 
(2) The contents of exercise

Although the percentage of the exercise habit was $61 \%$ at the time of first evaluation, by the second evaluation, it became $96 \%$ and almost all persons had added exercise to their lives. The content was shown in table 3. As for the exercise item, "walk," "swimming" and "stretching exercises" were given from the first to the third.

The average movement time per week was increasing by about 1.5 times after the second evaluation as compared with the first evaluation. Movement frequency is three to four days per week. It was admitted that the movement habit had improved dramatically by continuous attendance.

Table 3 Content of the Excises

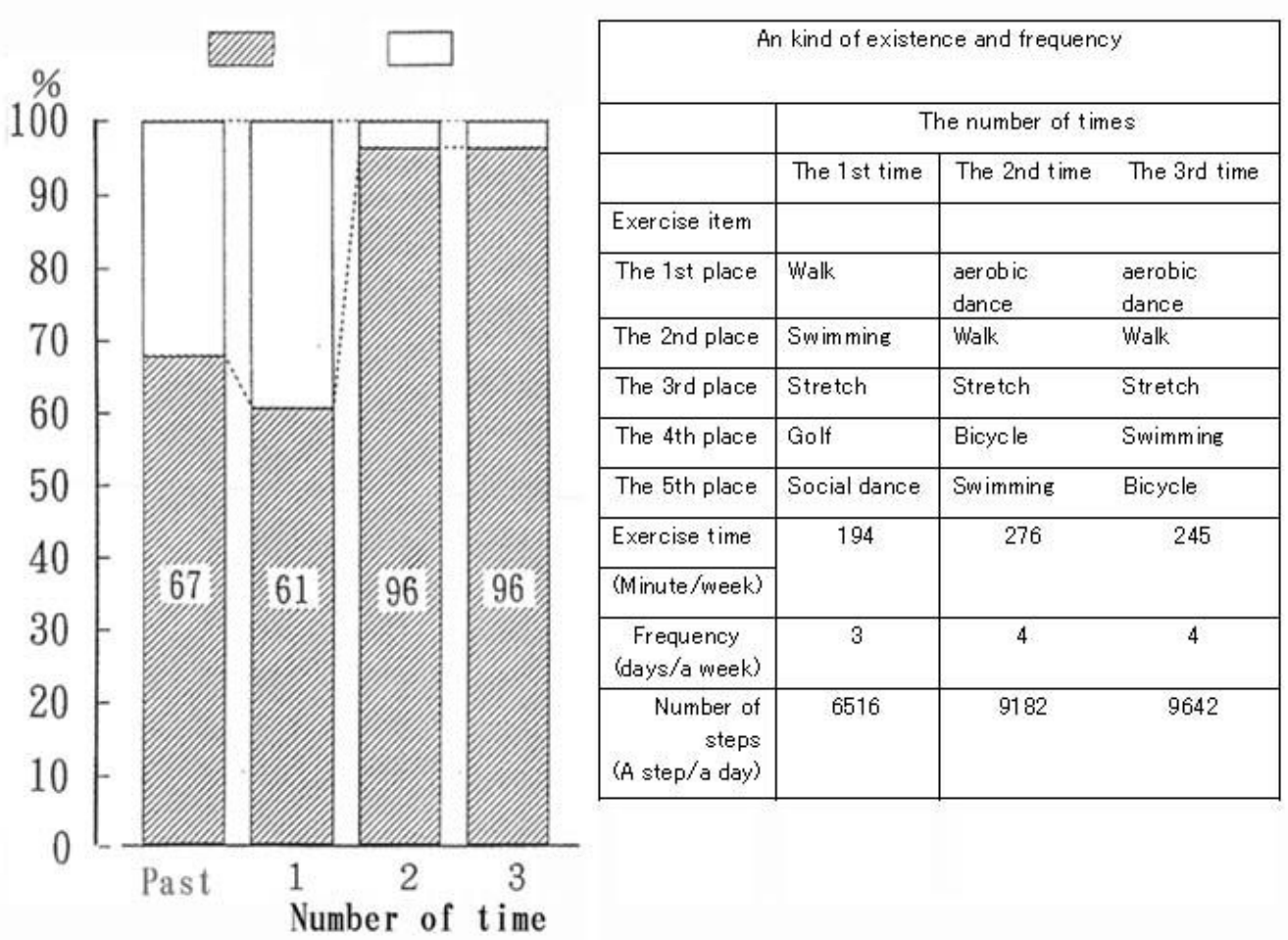

(3) Smoking and drinking habit

Although the persons with smoking habits was $14 \%$ of the whole and $50 \%$ of persons had an average of two to three days per week of drinking habits, those with both habits were not changing.

(4) Nutrition situation

The energy intake of the meal was $1700-1800 \mathrm{kcal}$ per day. This value is 5 to $15 \%$ over consumption requirements (the present standards of diet intake), and change was not admitted by the continuation subjects. However, the subjects' amount of body activities increased and percent of body fat decreased significantly. Especially when the calcium intake was $536 \mathrm{mg}$ and wasn't filling requirements, was improved after the first evaluation and was maintained.

\section{(5) Change of physical strength}

Exercise training in positive health class focuses on an improvement of aerobic ability. As shown in Fig 2, the maximal oxygen consumptions of subjects increase with each exercise session. The values of the second exercise session significantly increase effectiveness. In addition, the values to straighten posture and to stand on one leg with eyes closed increased significantly before the second session and the values of repetitive horizontal jump and of upper body forward flexion in long sitting position increased after the first session significantly. 
(6) Change of a blood lipid system

In the serum chemistry aspect, especially in changes of lipid systems as shown in Fig. 3, H DL cholesterol continued to increase after the first exercise session, showing a much higher value than before the first exercise session. Neutral fat dropped to a lower value. On the other hand, total cholesterol decreased during the exercise session, but it increased by the next exercise session. It repeated these patterns. There was a test subject whose total cholesterol was more that $230 \mathrm{mg}$ per $\mathrm{dl}$, but the index of arteriosclerosis changed in standard value. This is considered to be a major factor in the improvement of oxygen capability and the increase of HDL-cholesterol due to an increase in an exercise regimen.
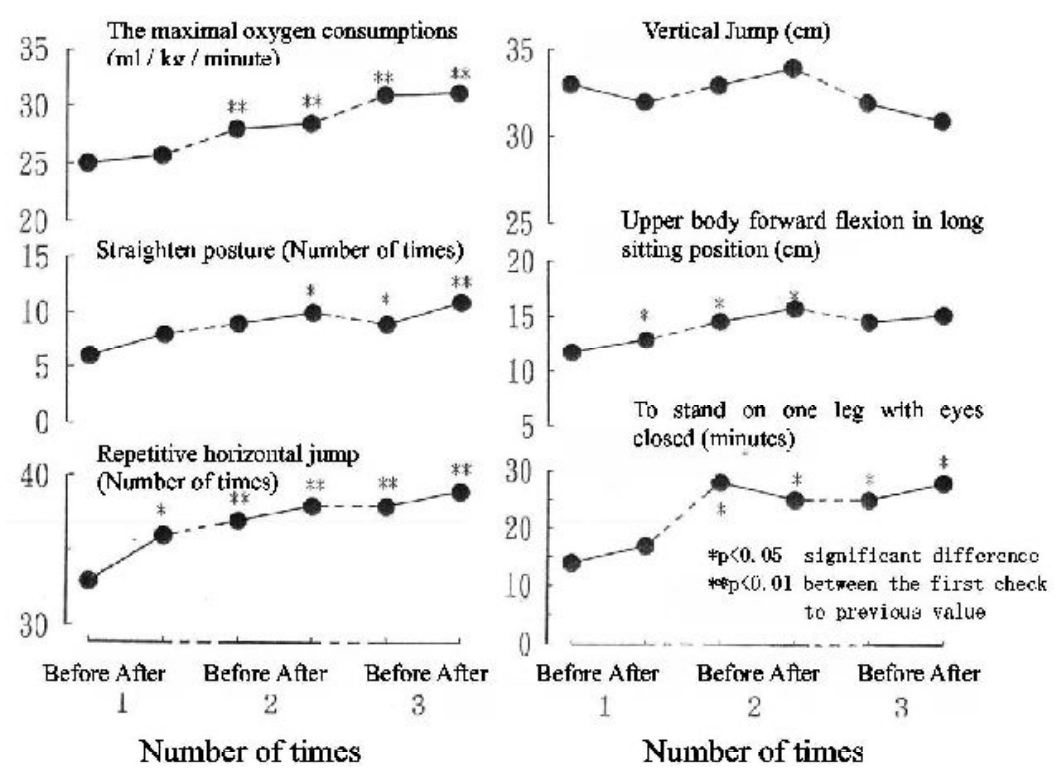

Fig. 2 Change of weight and body fat ratio

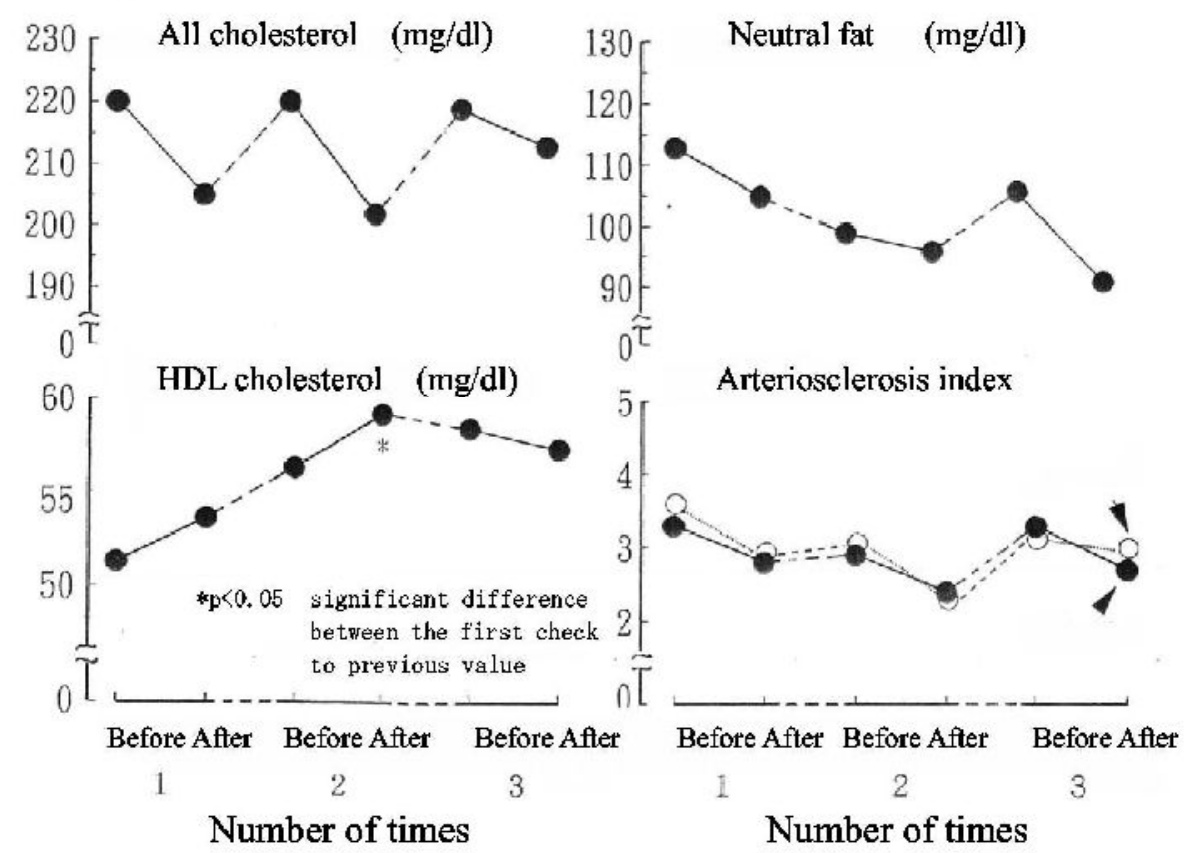

Fig. 3 Change of a blood lipid system 


\section{Conclusion}

In the objective area of this report, factors including residence, privacy, government and expert promotion of educational activity for health promotion by the developed health behavior determined by "ideal local-community" and achievement goals. 15), 16), 17) This time, authors used an empowerment technique when they planned, and made the process of establishment clear focusing on the result of middle-aged women's health care from the cycle of planning, enforcement and estimate. With understanding and cooperation of measurable subjects, they reaped the result of change of health activity by continuing education from the view of positive health. From this result, the effect was accepted in body composition, physical strength, and a blood lipid system item by continuing the positive health class. It is considered as the result of re-motivating to consider and perform the improvement of lifestyle, especially an exercise lifestyle for oneself by continuing the program. Having observed the accomplishments described above, using epidemiological methods which bring long term continuing results and QOL into view is important to promote estimates and adjustments for better education. We appreciate members of the Shiroyama-Cho health center, local residences who cooperated in the research and all testing subjects who participated in the positive health class.

\section{References}

1) Health promotion 1991. Supportive Environment WHO Sundsvall Statements, 1992.

2) The Public Health Service U.S.A., Healthy People 2000, 1994-1997.

3) Miyoko Hamano: Annual Report of Life culture museum of Tokyo Kasei Gakuin, 14: 85-96, 2004.

4) Wallerstein, N. and Bernstein, E : Empowerment education: Freire's ideas adapted to health education, Health Educ.Q, 15, 379-394(1988)

5) Wallerstein, N. Powerlessness, Empowerment, and health: Implications for health: promotion programs, Am. J . Public Health, 6. 197-205(1992).

6) Norie Anbai, the technique of community Empowerment, Ishiyaku Publishing Inc. 2005

7) Foundation of Health and an Improvement-of-Physical-Strength: the report of production movement of national health in the 21st century (Healthy Japan 21), 2000, 27-87

8) Toshihiro Iwanaga,: recommendation of the developed health behavior of community-based, Igaku-Shoin, 1995

9) Shuji Fujiuchi, et al: Manual of a health plan decision: For Practice of health polo motion, .Life science center, 2001

10) Nobuko Murayama, nutritional science magazine $\quad 61: 79-91,2003$

11) Aiko Kishimoto, et al. The individual health education intervention program in an area, and its evaluation, the 31 st Kanto farm village medical congress, 2003

12) Aiko Kishimoto, et al. In order to establish a healthy lifestyle, Proceedings of the 31st Kanto farm village medical congress, 51-52, 2002

13) Edited by health assessment workgroups, Health Education by Individual Guidance, Hoken-Dojin-Sha, 2002

14) Takeshi Kamishima, et al. Guidance of a new program, Hoken-Dojin-Sha, 2000

15) Akira Okayama, et al. : The Future and Past of Health Education, the, public health 66(7):443-437, 2002

16) The Concept and Practice of Emppowerment in a health promotion, nursing research, 30(5):453 - 458, 1997

17) Ayumi Yamada, the practice activities, research and subjects using Empowerment theory, Bulletin of the Science-of-nursing, Miyagi University 5(1):11 - 19, 2002 